\title{
On Optimization over the Efficient Set in Linear Multicriteria Programming ${ }^{1}$
}

\author{
R. Horst ${ }^{2}$, N.V. ThoAi ${ }^{3}$, Y. YAmamoto ${ }^{4}$, And D. ZenKE ${ }^{5}$
}

Communicated by G. Leitmann

\footnotetext{
${ }^{1}$ The research was partly done while the third author was visiting the Department of Mathematics, University of Trier with the support by the Alexander von Humboldt Foundation. He thanks the university as well as the foundation.

${ }^{2}$ Professor, Department of Mathematics, University of Trier, 54286 Trier, Germany

${ }^{3}$ Professor, Department of Mathematics, University of Trier, 54286 Trier, Germany

${ }^{4}$ Professor, Graduate School of Systems and Information Engineering, University of Tsukuba,

Tsukuba 305-8573, Japan

${ }^{5}$ Researcher, Graduate School of Systems and Information Engineering, University of Tsukuba,

Tsukuba 305-8573, Japan
} 


\section{Abstract:}

The efficient set of a linear multicriteria programming problem can be represented by a 'reverse convex constraint' of the form $g(z) \leq 0$, where $g$ is a concave function. Consequently, the problem of optimizing some real function over the efficient set belongs to an important problem class of global optimization called reverse convex programming. Since the concave function used in the literature is only defined on some set containing the feasible set of the underlying multicriteria programming problem, most global optimization techniques for handling this kind of reverse convex constraints can not be applied. The main purpose of our article is to present a method for overcoming this disadvantage. We construct a concave function which is finitely defined on the whole space and can be considered as an extension of the existing function. Different forms of the linear multicriteria programming problem are discussed, including the minimum maximal flow problem as an example.

Key words: Multicriteria optimization, optimization over the efficient set, global optimization, reverse convex constraint, minimum maximal flow problem. 


\section{Introduction}

Beginning with the work of Philip (Ref. 1), the problem of optimizing some real function over the efficient set of a linear multicriteria programming problem, abbreviated by (OEP), has received the attention of many authors and became one of the most important and interesting areas in multicriteria programming and global optimization, cf., e.g., Refs. 1-15. One of the most interesting approaches to handle Problem (OEP) is based on the representation of the efficient set using a 'reverse convex set', which is the complement of an open convex subset of the Euclidian space $\mathbb{R}^{n}$. Each reverse convex set is in principle described by a 'reverse convex constraint' of the form $g(z) \leq 0$, where $g$ is a concave function defined on $\mathbb{R}^{n}$. Up to the present, for such a representation of the efficient set of a linear multicriteria programming problem, one uses reverse convex constraints, in which the concave function $g$ can only be defined on some polyhedral set containing the feasible set of the underlying linear multicriteria programming problem. This fact has caused hindrances when applying existing methods for handling problems with reverse convex constraints. The reason is that the most promising algorithms for problems with reverse convex constraints are developed based on an assumption that the concave function $g$ must take finite values on a suitably large open set containing the feasible set, cf., e.g., Refs. 16-21. 
The main purpose of this article is to propose a way to represent the efficient set of a linear multicriteria programming problem by a reverse convex constraint, in which the concave function is finitely defined on the whole space. This concave function can be considered as an extension of the concave function used in the literature.

The reverse convex representation of the efficient set is discussed in the next section for the case that the feasible set of the underlying linear multicriteria programming problem is given by a system of inequalities. The case where the mentioned feasible set is described by equations is considered in Section 3. Section 4 deals with the minimum maximal flow problem, which is actually an example of the case discussed in Section 3. The article is completed with some conclusions in Section 5.

\section{Reverse Convex Representation of the Efficient Set}

\subsection{Reverse Convex Representation}

Let $Z$ be a polyhedral subset of $\mathbb{R}^{n}$ defined by

$$
Z=\left\{z \in \mathbb{R}^{n}: D z \leq d, z \geq 0\right\}
$$


where $D$ is an $m \times n$ matrix and $d \in \mathbb{R}^{m}$. For a given $p \times n$ matrix $C$ we consider the following multicriteria optimization problem

$$
\max \{C z: z \in Z\}
$$

A point $\bar{z} \in Z$ is said to be an efficient point of Problem (2) if there is no point $z$ of $Z$ such that $C z \geq C \bar{z}$ and $C z \neq C \bar{z}$. Let us denote the set of efficient points of Problem (2) by $Z^{E}$ and assume throughout this paper that

$$
Z \neq Z^{E} \neq \emptyset
$$

The optimization problem over the efficient set is then formulated as

$$
\min \left\{f(z): z \in Z^{E}\right\}
$$

with a given function $f$ defined on some suitable set containing $Z^{E}$.

We recall below some known useful characterizations of the efficient set $Z^{E}$ (cf., e.g., Refs. $1,15,22)$.

Proposition 1 Under Assumption (3) we obtain following characterizations of the efficient set: 
(a) $\bar{z}$ is an efficient point if and only if there is a positive vector $\lambda \in \mathbb{R}^{p}$ such that $\bar{z}$ solves the problem $\max \left\{\lambda^{T} C z: z \in Z\right\}$.

(b) $\bar{z}$ is an efficient point if and only if it solves the problem $\max \left\{\lambda^{T} C z: z \in Z, C z \geq C \bar{z}\right\}$ for each positive vector $\lambda \in \mathbb{R}^{p}$.

(c) Let $d^{i}, i=1, \cdots, m$ be the rows of the matrix $D$. Then $\bar{z} \in Z$ is an efficient point if and only if the following system (in variables $\lambda \in \mathbb{R}^{p}, \mu \in \mathbb{R}^{m}, \nu \in \mathbb{R}^{n}$ ) has a solution:

$$
\begin{aligned}
& C^{T} \lambda=D^{T} \mu-\nu \\
& \mu \geq 0 \text { and } \mu_{i}=0 \text { for } i \notin I(\bar{z}) \\
& \nu \geq 0 \text { and } \nu_{j}=0 \text { for } j \notin J(\bar{z}) \\
& \lambda \geq e,
\end{aligned}
$$

where $I(\bar{z})=\left\{i:\left(d^{i}\right)^{T} \bar{z}=d_{i}\right\}, J(\bar{z})=\left\{j: \bar{z}_{j}=0\right\}$, and $e=(1, \cdots, 1)^{T} \in \mathbb{R}^{p}$.

From Proposition 1(a), if we have a positive $\lambda \in \mathbb{R}^{p}$ such that Problem $\max \left\{\lambda^{T} C z: z \in Z\right\}$ has an optimal solution, then each optimal solution is an efficient point.

Conversely, from Proposition 1(c), if we have an efficient point $\bar{z}$, then, by computing a 
solution $(\bar{\lambda}, \bar{\mu}, \bar{\nu}) \in \mathbb{R}^{p+m+n}$ of System (S), we obtain a positive $\bar{\lambda} \in \mathbb{R}^{p}$ such that $\bar{z}$ is an optimal solution of Problem $\max \left\{\bar{\lambda}^{T} C z: z \in Z\right\}$.

Based on Proposition 1(b), letting $\lambda \in \mathbb{R}^{p}$ be a positive vector and

$$
\Omega=\left\{z \in \mathbb{R}^{n}: \exists y \in Z \text { such that } C y \geq C z\right\}
$$

one constructs a function $g_{\lambda}: \Omega \rightarrow \mathbb{R}$ by

$$
g_{\lambda}(z):=-\lambda^{T} C z+\max \left\{\lambda^{T} C y: y \in Z ; C y \geq C z\right\}
$$

The following result is also well-known (cf. e.g., Refs. 2, 15).

Proposition 2 For each positive vector $\lambda \in \mathbb{R}^{p}$ it holds that

(a) $g_{\lambda}(z)$ is finitely defined on the set $\Omega$.

(b) $g_{\lambda}(z)$ is a nonnegative piecewise linear concave function on $\Omega$.

(c) $z \in Z^{E}$ if and only if $z \in Z$ and $g_{\lambda}(z)=0$. 
Remark 1 By definition, the function $g_{\lambda}$ assumes finite values only on the set $\Omega$ defined in (5). For $z \notin \Omega$, the feasible set of the problem $\max \left\{\lambda^{T} C y: y \in Z ; C y \geq C z\right\}$ in (8) is empty, and therefore, $g_{\lambda}(z)$ is not finitely defined.

From Proposition 2, one can take any positive vector $\lambda \in \mathbb{R}^{p}$, e.g., $\lambda=e$, and formulate Problem (4) as

$$
\min \left\{f(z): z \in Z, g_{\lambda}(z) \leq 0\right\}
$$

Since the function $g_{\lambda}(z)$ is concave, the constraint $g_{\lambda}(z) \leq 0$ is usually called a reverse convex constraint, and Problem (7) belongs to the class of so called reverse convex programs. This problem class is well studied in the area of global optimization, cf., e.g., Refs. 16-21. However, the most promising algorithms for the reverse convex programs are based on an assumption that the concave function in the reverse convex constraint must take finite values on a suitable open set containing the set $Z$. As seen above, the function $g_{\lambda}$ does not fulfill this important assumption, so that a direct application of existing algorithms is not possible. In what follows we present a way to construct a concave function, which is defined on the whole space $\mathbb{R}^{n}$, so that by using it, algorithms for solving reverse convex programs can be applied. Moreover, since this function is finitely defined everywhere, it can provide some 
information about how far away a given point $x \in \mathbb{R}^{n}$ is from the efficient set.

To this purpose, letting $\bar{z} \in Z^{E}$ and $\bar{\lambda}$ be a positive vector such that $\bar{z}$ solves the problem $\max \left\{\bar{\lambda}^{T} C z: z \in Z\right\}$ (cf. Proposition 1), we define the function

$$
g_{\bar{\lambda}}(z)=-\bar{\lambda}^{T} C z+\max \left\{\bar{\lambda}^{T} C y: y \in Z, C y \geq C z\right\} .
$$

As discussed above, in principle, the function $g_{\bar{\lambda}}$ is only finitely defined on the set $\Omega$ given in (5). However, by using the above chosen vector $\bar{\lambda}$, we can construct an extension of $g_{\bar{\lambda}}$ on the whole space $\mathbb{R}^{n}$ as follows.

Since

$$
\max \left\{\bar{\lambda}^{T} C y: y \in Z ; C y \geq C z\right\} \leq \max \left\{\bar{\lambda}^{T} C y: y \in Z\right\}<+\infty
$$

it follows that the linear program

$$
\begin{array}{ll}
(Q(z)) \quad \max & \bar{\lambda}^{T} C y \\
\text { s.t. } & D y \leq d \\
& C y \geq C z \\
& \\
& y \geq 0
\end{array}
$$

has an optimal solution whenever it is feasible. On the other hand, we see that its dual problem, 


$$
\begin{array}{ll}
(R(z)) \quad \min \quad d^{T} \alpha-(C z)^{T} \beta \\
\text { s.t. } \quad D^{T} \alpha-C^{T} \beta \geq C^{T} \bar{\lambda} \\
& 0 \leq \alpha \\
& 0 \leq \beta,
\end{array}
$$

always has a nonempty feasible region, because from a solution $(\bar{\lambda}, \bar{\mu}, \bar{\nu})$ of System $(\mathrm{S})$ in Proposition $1(\mathrm{c})$, we obtain a feasible solution $(\alpha, \beta)=(\bar{\mu}, 0)$ of Problem $(R(z))$. Therefore, we see from the duality theorem in linear programming that Problem $(Q(z))$ is feasible if and only if Problem $(R(z))$ has an optimal solution.

Note that the feasible set of Problem $(R(z))$ does not depend on $z$. Let $V$ denote the set of all extreme points of the feasible set of this problem. Then there exist vectors $\bar{\alpha}$ and $\bar{\beta}$ such that

$$
V \subset\{(\alpha, \beta):(0,0) \leq(\alpha, \beta) \leq(\bar{\alpha}, \bar{\beta})\} \text {, and } \bar{\mu} \leq \bar{\alpha}
$$

An estimation of the vectors $\bar{\alpha}, \bar{\beta}$ will be discussed in the next subsection.

Using these vectors $\bar{\alpha}, \bar{\beta}$, we consider the following problem, denoted by $(\bar{R}(z))$, which arises from Problem $(R(z))$ by adding to it some upper bounding constraints. 


$$
\begin{array}{ll}
(\bar{R}(z)) \quad \min \quad d^{T} \alpha-(C z)^{T} \beta \\
\text { s.t. } & D^{T} \alpha-C^{T} \beta \geq C^{T} \bar{\lambda} \\
& 0 \leq \alpha \leq \bar{\alpha} \\
& 0 \leq \beta \leq \bar{\beta} .
\end{array}
$$

Based on Problem $(\bar{R}(z))$, we define a function $g: \mathbb{R}^{n} \rightarrow \mathbb{R}$ by

$$
g(z)=-\bar{\lambda}^{T} C z+t(z)
$$

where $t(z)$ is the optimal value of Problem $(\bar{R}(z))$ for each $z$.

The function $g$ defined in (10) is an extension of the function $g_{\bar{\lambda}}$ defined in (8) in the following sense.

Proposition 3 The function $g$ defined in (10) has following properties:

(a) $g(z)$ is well defined and finite at all points $z \in \mathbb{R}^{n}$.

(b) $g(z)$ is a piecewise linear concave function on $\mathbb{R}^{n}$.

(c) $g(z)=g_{\bar{\lambda}}(z)$ whenever $g_{\bar{\lambda}}(z)$ is finitely defined.

(d) $z \in Z^{E}$ if and only if $z \in Z$ and $g(z) \leq 0$. 
Proof. For each $z \in \mathbb{R}^{n}$, Problem $(\bar{R}(z))$ has an optimal solution, since its feasible set is nonempty and bounded. Furthermore, whenever Problem $(R(z))$ has a finite optimal value, it has an optimal solution contained in the set $V$. Thus, Problem $(\bar{R}(z))$ has the same optimal value as Problem $(R(z))$. From this, Assertions (a), (b) and (c) follow. The last assertion (d) follows from Assertion (c) of Proposition 2 and Assertion (c) of this proposition.

Remark 2 (a) The function $g$ can be zero outside $Z$, and hence $g(z)=0$ does not imply that $z \in Z^{E}$.

(b) The dual problem of $(\bar{R}(z))$ reads

$$
\begin{array}{cl}
(\bar{Q}(z)) \quad & \left(\bar{\lambda}^{T} C\right) y-\bar{\alpha}^{T} u-\bar{\beta}^{T} v \\
\text { s. t. } \quad & D y-u \leq d \\
C y+v \geq C z \\
y, u, v \geq 0 .
\end{array}
$$

Comparing Problems $(Q(z))$ and $(\bar{Q}(z))$, we see that $\bar{\alpha}$ and $\bar{\beta}$ can be considered as some kinds of control parameters for the construction of the extension function. 


\subsection{Bounding the Set of Vertices}

We present here a way to estimate the vectors $\bar{\alpha}$ and $\bar{\beta}$ for bounding the vertex set $V$ of the feasible set of Problem $(\mathrm{R}(\mathrm{z}))$.

Proposition 4 Assume that the elements

$$
\begin{aligned}
& d_{i j},(i=1, \cdots, m ; j=1, \cdots, n) \text { and } \\
& c_{i j},(i=1, \cdots, p ; j=1, \cdots, n)
\end{aligned}
$$

of the matrices $D$ and $C$, respectively, are all integral. Define

$$
\begin{aligned}
& M=\max \left\{\max \left\{\left|d_{i j}\right|: i=1, \cdots, m ; j=1, \cdots, n\right\}, \max \left\{\left|c_{i j}\right|: i=1, \cdots, p ; j=1, \cdots, n\right\}\right\} \\
& \Lambda=\max \left\{\left|\bar{\lambda}_{i}\right|: i=1, \cdots, p\right\}
\end{aligned}
$$

Then a common upper bound, $\sigma$, for all components $\bar{\alpha}_{i}(i=1, \cdots, m)$, and $\bar{\beta}_{i}(i=1, \cdots, p)$ can be given by

$$
\sigma=n(n-1)^{\frac{1}{2}(n-1)} p M^{n} \Lambda
$$

Proof. Let $\gamma=(\alpha, \beta) \in V$. Then each positive component $\gamma_{j_{i}}$ is computed by

$$
\gamma_{j_{i}}=\left(B^{-1}\left(C^{T} \bar{\lambda}\right)\right)_{i}
$$

where $B$ is a nonsingular $n \times n$ submatrix of the $n \times(m+p+n)$ matrix $\left(D^{T},-C^{T},-I\right)$ with $I$ being the $n \times n$ identity matrix. Let $B_{j i}$ be the matrix obtained from $B$ by deleting the 
$j$-th row and the $i$-th column. Then $B^{-1}=\frac{1}{\operatorname{det} B} B^{*}$, where each element $b_{i j}^{*}$ of the matrix $B^{*}$ is determined by $b_{i j}^{*}=(-1)^{i+j} \operatorname{det} B_{j i}$. From the well-known Hadarmard's inequality, it follows that

$$
\left|\operatorname{det} B_{j i}\right| \leq \prod_{k=1}^{n-1}\left((n-1) M^{2}\right)^{\frac{1}{2}}=(n-1)^{\frac{1}{2}(n-1)} M^{n-1}
$$

Therefore, we have

$$
\begin{aligned}
\gamma_{j_{i}}=\left|\gamma_{j_{i}}\right|=\left|\left(B^{-1}\left(C^{T} \bar{\lambda}\right)\right)_{i}\right| & =\frac{1}{|\operatorname{det} B|}\left|\sum_{k=1}^{n} b_{j_{i}, k}^{*}\left(C^{T} \bar{\lambda}\right)_{k}\right| \\
& \leq \frac{1}{|\operatorname{det} B|} \sum_{k=1}^{n}\left|b_{k, j_{i}}\right|\left|\left(C^{T} \bar{\lambda}\right)_{k}\right| \\
& \leq \frac{1}{|\operatorname{det} B|} n(n-1)^{\frac{1}{2}(n-1)} M^{n-1} p M \Lambda \\
& \leq n(n-1)^{\frac{1}{2}(n-1)} p M^{n} \Lambda .
\end{aligned}
$$

\section{Multicriteria Optimization with Equality Constraints}

In this section, we consider multicriteria optimization problems of the form

$$
\max \{\bar{C} x: A x=b, x \geq 0\}
$$


where $\bar{C}$ and $A$ are matrices of dimension $p \times q$ and $m \times q$, respectively, and $b \in \mathbb{R}^{m}$. We denote the feasible set of this problem by $X$, i.e.,

$$
X=\{x: A x=b ; x \geq 0\}
$$

and the set of efficient points by $X^{E}$. The optimization problem to be considered is

$$
\min \left\{h(x): x \in X^{E}\right\}
$$

with $h$ being a given real function on $\mathbb{R}^{q}$.

We assume without loss of generality that $\operatorname{rank} A=m$ and $A=(B N)$, where $B$ is a regular $m \times m$ submatrix. Usually, $B$ and $N$ are called basic and nonbasic matrices, respectively, of the system $A x=b$. Thus, the dimension of $X$ is then $n=q-m$, and Problem (12) in $\mathbb{R}^{q}$ can be transformed into a problem in $\mathbb{R}^{n}$ as follows.

According to the structure of the matrix $A$, we consider the vector $x$ and the matrix $\bar{C}$ as $x^{\top}=\left(x_{B}^{\top}, x_{N}^{\top}\right)$ and $\bar{C}=\left(\bar{C}_{B}, \bar{C}_{N}\right)$, and define on $\mathbb{R}^{n}$ the polyhedral set

$$
X_{N}=\left\{x_{N} \in \mathbb{R}^{n}: B^{-1} N x_{N} \leq B^{-1} b ; x_{N} \geq 0\right\}=\left\{x_{N} \in \mathbb{R}^{n}: T x_{N} \leq t\right\},
$$

where $T$ and $t$ are a $q \times n$ matrix and $q$-dimensional vector, respectively, defined by

$$
T=\left(\begin{array}{c}
B^{-1} N \\
-I
\end{array}\right), \quad t=\left(\begin{array}{c}
B^{-1} b \\
0
\end{array}\right)
$$


with $I$ being the identity matrix.

Below we give the elementary relationship between $X_{N}$ and $X$ with a simple proof. For a polyhedral set $P$ let $V(P)$ denote the set of extreme points of $P$.

Proposition 5 It holds that

(a) $x=\left(x_{B}^{\top}, x_{N}^{\top}\right)^{\top} \in X \Leftrightarrow x_{N} \in X_{N}, x_{B}=B^{-1} b-B^{-1} N x_{N}$;

(b) $x=\left(x_{B}^{\top}, x_{N}^{\top}\right)^{\top} \in V(X) \Leftrightarrow x_{N} \in V\left(X_{N}\right), x_{B}=B^{-1} b-B^{-1} N x_{N}$.

\section{Proof.}

(a) We have

$$
\begin{aligned}
\left(x_{B}^{\top}, x_{N}^{\top}\right)^{\top} \in X & \Leftrightarrow B x_{B}+N x_{N}=b, x_{B} \geq 0, x_{N} \geq 0 \\
& \Leftrightarrow x_{B}=B^{-1} b-B^{-1} N x_{N}, x_{B} \geq 0, x_{N} \geq 0 \\
& \Leftrightarrow x_{B}=B^{-1} b-B^{-1} N x_{N}, T x_{N} \leq t \\
& \Leftrightarrow x_{B}=B^{-1} b-B^{-1} N x_{N}, x_{N} \in X_{N} .
\end{aligned}
$$


(b) follows from the following equivalences:

$$
\begin{aligned}
\left(x_{B}^{\top}, x_{N}^{\top}\right)^{\top} \in X \backslash V(X) \Leftrightarrow & \exists \operatorname{distinct}\left(\left(x_{B}^{1}\right)^{\top},\left(x_{N}^{1}\right)^{\top}\right)^{\top},\left(\left(x_{B}^{2}\right)^{\top},\left(x_{N}^{2}\right)^{\top}\right)^{\top} \in X \text { such that } \\
& \left.\left(x_{B}^{\top}, x_{N}^{\top}\right)=\frac{1}{2}\left(\left(x_{B}^{1}\right)^{\top},\left(x_{N}^{1}\right)^{\top}\right)+\left(\left(x_{B}^{2}\right)^{\top},\left(x_{N}^{2}\right)^{\top}\right)\right) \\
\Leftrightarrow & \exists \text { distinct } x_{N}^{1}, x_{N}^{2} \in X_{N} \text { such that } \\
& x_{N}=\frac{1}{2}\left(x_{N}^{1}+x_{N}^{2}\right), x_{B}=\frac{1}{2}\left(x_{B}^{1}+x_{B}^{2}\right)=B^{-1} b-B^{-1} N x_{N} \\
\Leftrightarrow & x_{N} \in X_{N} \backslash V\left(X_{N}\right), x_{B}=B^{-1} b-B^{-1} N x_{N} .
\end{aligned}
$$

The first and third equivalences are obtained by the definition of extreme points. The second equivalence follows from part (a) of this proposition.

Based on the above observation, using the representation of the matrix $\bar{C}$ as $\bar{C}=\left(\bar{C}_{B}, \bar{C}_{N}\right)$, we can rewrite the objective functions of the multicriteria problem (11) in the form

$$
\bar{C} x=\left(\bar{C}_{B}, \bar{C}_{N}\right)\left(x_{B}^{T}, x_{N}^{T}\right)^{T}=\bar{C}_{B} B^{-1} b+(-\bar{C} T) x_{N}
$$

so that Problem (11) in $\mathbb{R}^{q}$ reduces to a problem of the form (1)-(2) in $\mathbb{R}^{n}$ :

$$
\max \left\{(-\bar{C} T) x_{N}:\left(B^{-1} N\right) x_{N} \leq B^{-1} b, x_{N} \geq 0\right\}
$$

Moreover, we have the following relationship between the efficient sets of the two problems. 
Proposition 6 Denote by $X_{N}^{E}$ the set of all efficient solutions of Problem (16). Then it holds that

$$
x=\left(x_{B}^{\top}, x_{N}^{\top}\right)^{\top} \in X^{E} \Leftrightarrow x_{N} \in X_{N}^{E} \text { and } x_{B}=B^{-1} b-B^{-1} N x_{N} .
$$

Proof. From Proposition 5(a) and the definition of efficient solutions, it follows that

$$
\begin{aligned}
x=\left(x_{B}^{\top}, x_{N}^{\top}\right)^{\top} \in X^{E} \Leftrightarrow & \left(x_{B}^{\top}, x_{N}^{\top}\right)^{\top} \in X, \nexists\left(y_{B}^{\top}, y_{N}^{\top}\right)^{\top} \in X \text { such that } \\
& \left(C_{B}, C_{N}\right)\left(y_{B}^{\top}, y_{N}^{\top}\right)^{\top} \geq\left(C_{B}, C_{N}\right)\left(x_{B}^{\top}, x_{N}^{\top}\right)^{\top}, \\
& \left(C_{B}, C_{N}\right)\left(y_{B}^{\top}, y_{N}^{\top}\right)^{\top} \neq\left(C_{B}, C_{N}\right)\left(x_{B}^{\top}, x_{N}^{\top}\right)^{\top} \\
\Leftrightarrow & x_{N} \in X_{N}, x_{B}=B^{-1} b-B^{-1} N x_{N}, \\
& \nexists\left(y_{B}^{\top}, y_{N}^{\top}\right)^{\top} \in X: y_{N} \in X_{N}, y_{B}=B^{-1} b-B^{-1} N y_{N} \text { such that } \\
& (-C T) y_{N} \geq(-C T) x_{N},(-C T) y_{N} \neq(-C T) x_{N} \\
\Leftrightarrow & x_{N} \in X_{N}^{E} \text { and } x_{B}=B^{-1} b-B^{-1} N x_{N} .
\end{aligned}
$$

Remark 3 Setting $z=x_{N}, C=-\bar{C} T, D=B^{-1} N$ and $d=B^{-1} b$ we obtain a problem of the form (2) from Problem (16). Moreover, under the assumption that all elements of the matrices $\bar{C}$ and $A$ in Problem (11) are integral, we obtain the assumption that all elements of matrices $C$ and $D$ in Problem (2) are integral, so that Proposition 4 can be applied for the estimation of vectors $\bar{\alpha}$ and $\bar{\beta}$ as discussed in Section 2. 
By the above transformation, the objective function $h$ on $\mathbb{R}^{q}$ in Problem (12) is then transformed to a function $f$ on $\mathbb{R}^{n}$ :

$$
f\left(x_{N}\right)=h\left(B^{-1} b-B^{-1} N x_{N}, x_{N}\right) .
$$

Note that if $h$ is a convex/concave function on $\mathbb{R}^{q}$, then $f$ is convex/concave as well on $\mathbb{R}^{n}$. In particular, if the function $h(x)$ is linear given by $h(x)=h^{T} x$ with $h \in \mathbb{R}^{q}$, then by using the partition $h^{T}=\left(h_{B}^{T}, h_{N}^{T}\right)$, Problem (12) reduces to

$$
\min \left\{\left(h_{N}^{T}-h_{B}^{T} B^{-1} N\right) x_{N}: x_{N} \in X_{N}^{E}\right\}
$$

\section{Minimum Maximal Flow Problem}

As an example for the problem class discussed in Section 3, we consider a directed network with a single source node and a single sink node. The node-arc incidence matrix and the vector of arc capacities are denoted by $E$ and $c$, respectively. A flow $f$ is called a maximal flow if it is an efficient point of the following multicriteria network flow problem:

$$
\max \{I f: E f=0,0 \leq f \leq c\}
$$

where the identity matrix $I$, the matrix $E$ and vectors $f, c$ are of appropriate sizes. 
Let $F$ and $F^{E}$ denote the feasible set and the efficient set, respectively, of Problem (19), and according to $f$, let $h$ be a vector defined by

$$
h_{j}=\left\{\begin{aligned}
+1 & \text { if arc } j \text { leaves the source node } \\
-1 & \text { if arc } j \text { enters the source node } \\
0 & \text { otherwise. }
\end{aligned}\right.
$$

Then the minimum maximal flow problem considered in Refs. 23-26 is formulated as

$$
\min \left\{h^{T} f: f \in F^{E}\right\} .
$$

Using the notations

$$
x=\left(\begin{array}{c}
f \\
s
\end{array}\right), \quad \bar{A}=\left(\begin{array}{cc}
E & 0 \\
I & I
\end{array}\right), \quad \bar{b}=\left(\begin{array}{l}
0 \\
c
\end{array}\right), \quad \bar{C}=\left(\begin{array}{ll}
I & 0
\end{array}\right),
$$

where $s$ is the vector of slack variables for the Constraint $f \leq c$, we can rewrite Problem (19) in the form

$$
\max \{\bar{C} x: \bar{A} x=\bar{b}, x \geq 0\}
$$

Finally, removing all linear dependent equations of System $\bar{A} x=\bar{b}$, we obtain from Problem (21) a multicriteria network flow problem, which has the form of the problem considered in Section 3. 


\section{Conclusions}

We have presented a method for the construction of a concave function finitely defined on the whole space, which can be used to represent the efficient set of a linear multicriteria programming problem by a reverse convex constraint. This allows to handle some optimization problems over the efficient set by applying standard techniques in reverse convex programming. 


\section{References}

1. PhiliP, J., Algorithms for the Vector Maximization Problem, Mathematical Programming, Vol.2, p. 207-229, 1972.

2. Benson, H.P., Optimization over the Efficient Set, Journal of Mathematical Analysis and Applications, Vol. 98, p. 562-580, 1984.

3. Benson, H.P., An All-Linear Programming Relaxation Algorithm for Optimizing over the Efficient Set, Journal of Global Optimization, Vol. 1, p. 83-104, 1991.

4. Benson, H.B. and Lee, D., Outcome-based Algorithm for Optimizing over the Effcient Set of a Bicriteria Linear Programming Problem, Journal of Optimization Theory and Applications, Vol. 88, p. 77-105, 1996.

5. Bolintineanu, S., Minimization of a Quasiconcave Function over an Efficient Set, Mathematical Programming, Vol. 61, p. 89-110, 1993.

6. Dauer, J.P., and Fosnaugh, T.A., Optimization over the Efficient Set, Journal of Global Optimization, Vol. 7, p. 261-277, 1995.

7. Le-Thi H.A., Pham D.T., and MuU, L.D., Numerical Solution for Optimization over the Efficient Set by D.C. Optimization Algorithms, Operations Research Letters, Vol. 19, p. 117-128, 1996.

8. Muu, L.D., and Luc, L.T., On Equivalence between Convex Maximization and Optimization over the Efficient Set, Vietnam Journal of Mathematics, Vol. 24, p. 439-444, 1996. 
9. Horst R., and ThoAi N.V., Utility Function Programs and Optimization over the Eficient Set in Multiple Objective Decision Making, Journal of Optimization Theory and Applications, Vol. 92, p. 469-486, 1997.

10. Horst R., and ThoAi N.V., Maximizing a Concave Function over the Efficient or Weakly-Eficient Set, European Journal of Operations Reseach, Vol. 117, p. 239-252, 1999.

11. ThoAi, N.V., A class of optimization problems over the efficient set of a multiple criteria nonlinear programming problem, European Journal of Operational Research, Vol. 122, p. 58-68, 2000.

12. Thoni, N.V., Conical Algorithm in Global Optimization for Optimizing over Efficient Sets, Journal of Global Optimization, Vol. 18, p. 321-336, 2000.

13. Thoni, N.V., Convergence and Application of a Decomposition Method Using Duality Bounds for Nonconvex Global Optimization, Journal of Optimization Theory and Applications, Vol. 113, p.165-193, 2002.

14. Le-Thi H.A., Pham D.T. and Thoai, N.V., Combination between Global and Local Methods for Solving an Optimization Problem over the Efficient Set, European Journal of Operational Research, Vol. 142, p. 258-270, 2002.

15. Yамамото, Y., Optimization over the Efficient Set: Overview, Journal of Global Optimization, Vol. 22 , p. 285-317, 2002. 
16. Hillestad, R.J., and Jacobsen, S.E., Reverse Convex Programming, Applied Mathematics and Optimization, Vol. 6, p. 63-78, 1980.

17. Thoni, N.V., Canonical D.C. Programming Techniques for Solving a Convex Program with an Additional Constraits of Multiplicative Type, Computing, Vol. 50, p. 241-253, 1993.

18. Horst, R., and Thoni, N.V., Constraint Decomposition Algorithms in Global Optimization, Journal of Global Optimization, Vol. 5, p. 333-348, 1994.

19. Horst, R., and Tuy, H., Global Optimization: Deterministic Approaches, 3rd edition, Springer, Berlin, 1996.

20. Horst, R., Pardalos, P.M., and Thoai, N.V., Introduction to Global Optimization, 2nd Edition, , Kluwer, Dordrecht, The Netherlands, 2000.

21. Horst R., and Thoai N.V., D.C. Programming: Overview, Journal of Optimization Theory and Applications, Vol. 103, p. 1-43, 1999.

22. Yu, P.L., Multiple Criteria Decision Making: Concepts, Techniques, and Extensions, Plenum, New York, 1985.

23. Shi, J., and Yамамото, Y., A Global oOptimization Method for Minimum Maximal Flow Problem, Acta Mathematica Vietnamica, Vol. 22, p. 271-287, 1997.

24. Gotoh, J., Thoni, N.V., and Yamamoto, Y., Global Optimization Method for Solving the Minimum Maximal Flow Problem, Optimization Methods and Software, 
Vol. 18, p. 395-415, 2003.

25. Shigeno, M., Takahashi, I., and Yamamoto, Y., Minimum Maximal Flow Problem - an Optimization over the Efficient Set, Journal of Global Optimization, Vol. 25, p. $425-443,2003$.

26. Yamamoto, Y., and Zenke, D., Cut and Split Method for the Minimum Maximal Flow Problem, Pacific Journal of Optimization, Vol. 1, p. 387-404, 2005. 